\title{
CHROMOSPHERIC TELESCOPE OF BAIKAL ASTROPHYSICAL OBSERVATORY. NEW LIGHT
}

\author{
V.I. Skomorovsky, G.I. Kushtal, L.S. Lopteva, V.A. Proshin, V.D. Trifonov, S.A. Chuprakov, \\ V.A. Khimich
}

Institute of Solar-Terrestrial Physics SB RAS, Irkutsk, Russia, skoal@iszf.irk.ru

A chromospheric telescope is an important instrument for synoptic observations and solar research. After several decades of observations with the chromospheric telescope at the Baikal Astrophysical Observatory, a need arose to improve the characteristics of this telescope and filter. A new reimaging lens to produce full-disk solar images $18 \mathrm{~mm}$ in diameter at the CCD camera Hamamatsu C-124 with a $36 \times 24 \mathrm{~mm}$ detector $(4000 \times 2672$ pixels $)$ was designed and manufactured to replace the out-ofoperation $50 \times 50 \mathrm{~mm}$ Princeton Instruments camera. A contrast interference blocking filter and new calcite and quartz crystal plates were made and installed instead of damaged ones in the Ho birefringent filter (BF), manufactured by Bernhard Halle Nachfl. The optical immersion in the filter was changed. All telescope optics was cleaned and adjusted. We describe for the first time the design features and their related BF passband tuning. The wavefront interferograms of optical elements and telescope as a whole show that the wavefront distortion of the optical path is within $0.25 \lambda$. The BF and prefilter spectral parameters provide high-contrast monochromatic images. Besides, we give examples of solar chromospheric images in the $\mathrm{H} \alpha$ line core and wing.

Keywords: Solar telescope, birefringent filter, interferometric measurements.

\section{INTRODUCTION}

In 1980, SibIZMIR devised and manufactured the first domestic telescope for H $\alpha$ filter observation of the chromosphere of the full solar disk with spatial resolution of about 1" [Banin et al., 1982].

Main parameters of the chromospheric telescope and filter

\begin{tabular}{|l|l|}
\hline Diameter of the main lens & $180 \mathrm{~mm}$ \\
\hline Equivalent focal distance & $5432 \mathrm{~mm}$ \\
\hline Non-vignetted field of view & $34 \mathrm{arcmin}$ \\
\hline Solar image diameter & $50 \mathrm{~mm}$ \\
\hline Birefringent filter, manufacturer & Bernhard Halle Nachfl. GmbH \\
\hline Passband wavelength & $\lambda 6563 \AA$ \\
\hline Passband half-width & 1 or $0.5 \AA$ \\
\hline Passband shifts within & $\pm 1 \AA$. \\
\hline
\end{tabular}


Over a period from 1981 to $1999,50 \mathrm{~mm}$ solar images were taken using an $80 \mathrm{~mm}$ film. The film archive is kept in the Institute of Solar-Terrestrial Physics SB RAS. In 2000-2002, the images were captured by a Princeton Instruments CCD camera with a $50 \times 50 \mathrm{~mm}$ matrix detector $(2048 \times 2048$ pixels $)$ [ftp://ftp.iszf.irk.ru/h_alpha]. Since 2004, because of the camera failure, the images were obtained manually with a Konica Minolta DiMAGE A2 8MP Digital Camera (3264×2448 pixels). The small camera view (about $17 \mathrm{~mm}$ ) allowed us to observe only individual active regions. To take full-disk images by a new automatic Hamamatsu C9300-124 CCD camera with a $36 \times 24 \mathrm{~mm}$ detector $(4000 \times 2672$ pixels), we temporally installed a commercial lens that produced $18 \mathrm{~mm}$ solar images. However, it did not provide high-quality images; hence the need for a new reimaging lens. Moreover, in images there emerged areas with different center-to-limb brightness, whose sharp jump pointed to instrumental origin, as well as dark points in the field of view and drop-shaped defects at the field edge. They had been caused by defects arising in the optical stack of the birefringent filter (BF) and in the prefilter. To correct the defects, we had to repair and change BF polarization elements. Despite the relatively clean Baikal atmosphere, the telescope optics over the 35-year period of year-round operation were covered with dust from the nearest buildings under construction and with soot from forest fires. We had to completely dissemble, clean the teleobjective and other optics of the telescope, and readjust the entire telescope.

\section{DESCRIPTION OF THE TELESCOPE}

The telescope and its optical scheme are shown in Figure 1. One of the conditions for successful operation of the telescope - filter complex was the compliance of its optical scheme for image construction with BF installation requirements largely in order to maintain the emission monochromaticity, specified by BF parameters, and to provide the required resolution over the entire solar disk.

Particular original design solutions for optical elements and some other essential unites of the chromospheric H $\alpha$ telescope are presented in [Klevtsov, Trifonov, 1980].

$\mathrm{BF}$ - the most important element of the chromospheric telescope - is a unique instrument that costs as much or even more than the telescope. The clear aperture of the BF optical stack is $28 \mathrm{~mm}$; the length, $190 \mathrm{~mm}$; the filter field of view, $\pm 2.5^{\circ}$ with allowable passband shift of $0.05 \AA$. These parameters suppress appetite for installation of the filter into a large aperture telescope for full-disk observation with the highest, less than 1", resolution [Klevtsov, 1984]. It was just this filter for which we calculated the optical scheme and manufactured the telescope to capture full-disk monochromatic solar images at spatial resolution of about 1 " with allowance made for resolution characteristics of emission receivers (motion picture films, CCD matrix). 

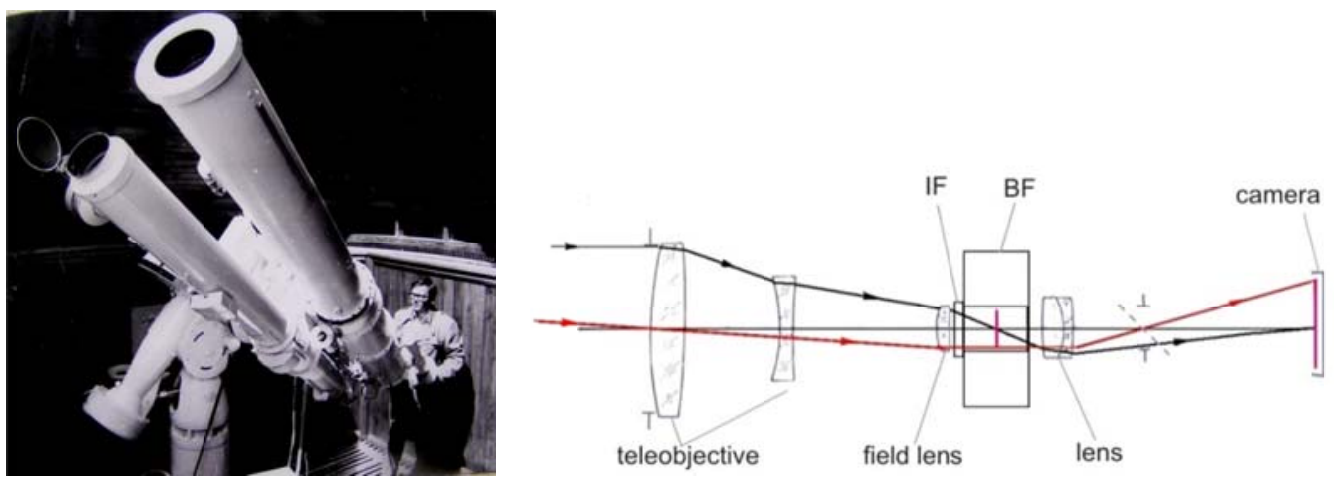

Figure 1. Chromospheric telescope of the Baikal Astrophysical Observatory and its optical scheme

We did not take on an inverse task to work out a filter for the finished telescope: in 1970-80s manufactures sought to design $\mathrm{BF}$ with extreme characteristics determined by currently available filter schematic solutions, birefringent crystals, and by processing and control capabilities. Large homogeneous calcite crystals required for large aperture filters are rare in nature and if available it is unreasonable to cut them for a medium-quality filter. The crystals may be kept in reserve until new schemes and technologies have been developed.

An interference filter (IF), a prefilter comprised of a metal - dielectric - metal (MDM) heat filter and a narrowband interference filter, transmits light only in a narrow spectral region with a central wavelength and reflects all the emission back through the telescope lens. Inside illumination of lenses by light reflected from the filter balances the outside heat flux and decreases temperature gradients in lenses.

Before the "first light" of the chromospheric telescope, we examined spectral characteristics and wavefront of BF and IF. Spectral characteristics of BAO BF were similar to those reported for the Bernhard Halle filter of the Sayan Solar Observatory [Skomorovsky, 1967]. In the crystal stack and BAO BF, we revealed wavefront distortions. They were diminished by a special laboratory-processed transparent plate that had been optically polished and retouched to compensate these distortions. The plate was installed outside of the BF crystal stack.

Teleobjective and field lens. The calculation of the optical scheme and the choice of lens design were limited to the use of a long focal-length lens: the telescope should be no more than $4 \mathrm{~m}$ long to fit within the pavilion dome. The short length of the objective unit of the telescope is due to the two-component teleobjective. A principally new idea was to install the field lens in front of the primary image to shorten the telescope tube [Banin et al., 1983a]. The focal distance of the field lens is equal to its distance from the image of pupil created by the negative component of the lens. The field lens transfers the pupil image to infinity and forms in the middle of the filter an intermediate solar image $20 \mathrm{~mm}$ in diameter in the telecentric beam path. This provides homogeneous spectral composition of all image points.

The telescope is designed to produce a high-quality image in the focal plane only in one wavelength; and this beneficial feature enables us to use for the teleobjective only two single-lens (uncemented) 
components, positive and negative, making them from one material and with radii of curvature that are equal in size but different in sign. The latter is very favorable for the mutual control of radii of curvature and lens surface shape when manufactured.

The lens reconstructs and enlarges a solar image so as not to lose the resolution expected of the telescope on film and then on matrix. If we accept that the actual resolution of film coating with relatively high resolution of 200 line/mm for low-contrast chromospheric structures is 30 line $/ \mathrm{mm}$, a solar image should be no less than $50 \mathrm{~mm}$. This roughly corresponds to the resolution of 1.3 ".

In the telescope scheme, the problems of control and adjustment have been successfully solved: the main lens and zooming lens were corrected for spherical aberration, thus providing independent control of each component in the autocollimation scheme with a plane mirror. We virtually eliminated the entire telescope system from spherical aberration, coma, and astigmatism. The tolerances for radii, thicknesses, inter-lens distances, and refractive indices of lenses are quite low. High tolerances exist for decentration of teleobjective, i.e. for wedge, rotation, and displacement of the lenses perpendicular to the optical axis [Klevtsov, Trifonov, 1980]. Adjustment of lenses in the telescope (tilt, displacement of lenses) was controlled using interference images in the lenses with the aid of a laser. The wavefront of the teleobjective and telescope after the first assembly with the filter optical stack simulator, which was installed instead of $\mathrm{BF}$ and transmitted laser light, was tested in the autocollimation scheme. Figure 2 shows wavefront interferograms of the teleobjective and telescope.

Observational materials on the solar chromosphere obtained with the telescope, their processing and interpretation are reported in research papers and theses by ISTP SB RAS and other researchers [Banin et al., 1983b; Banin et al., 1986; Borovik, Myachin, 2002, 2010; Golovko et al., 2002; Batmunkh et al., 2011; Borovik et al., 2014; Konyayev et al., 2015].

\section{MODERNIZATION OF THE TELESCOPE IN 2015}

Since the CCD camera was changed to another one with a matrix half as large, we had to calculate and make a new reimaging lens because the temporary commercial lens in use did not produce a highquality image. Fading of film coatings of the MDM heat filter, installed before BF, in the solar beam caused image brightness to vary across the field of view. It was immediately obvious that this was due to inhomogeneity of the prefilter passband.

In addition, we discovered defects in BF polarization elements - cement defects and cracks. The chromospheric telescope operating for many years, its optics was brought out of center, the filter appeared to be displaced, and edge defects of its optical stack also appeared within the field of view. 

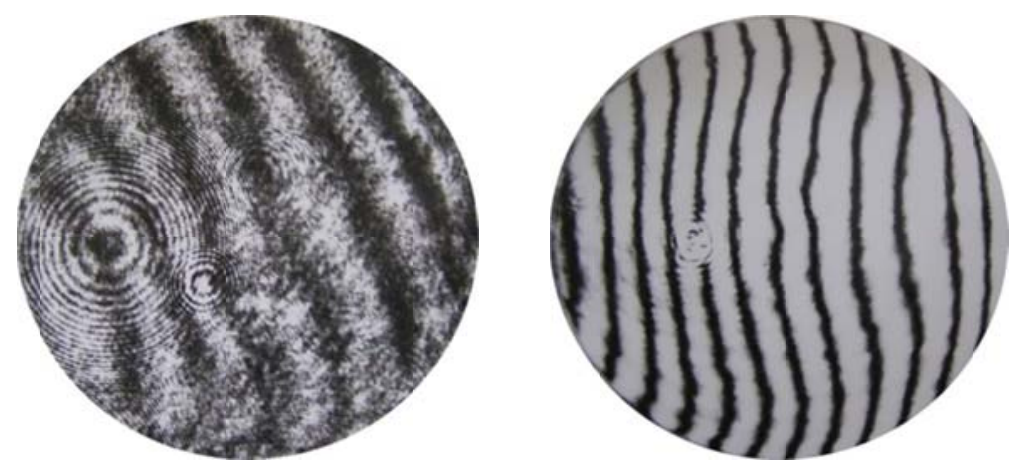

Figure 2. Wavefront interferogram of the teleobjective and telescope [Klevtsov, 1987]. The wavefront of the telescope is close to the theoretical limit

\section{BIREFRINGENT FILTER}

The company Bernhard Halle (West Berlin) has produced about 200 Lyot-Ohman filters [Bhatnagar, Livingston, 2005]. In 1960s, Astrocouncil of the USSR Academy of Sciences received ten H $\alpha$ filters and distributed them among USSR observatories: IZMIRAN and SibIZMIR observatories, Crimean Astrophysical Observatory (AO), Mountain Station of the Central Astronomical Observatory, Ussuriysk AO, Tashkent AO, and Ukraine MAO in Goloseyevo. Studies of B. Halle filters [Skomorovsky, 1967] revealed that their transmittance was not less than $10 \%$ because the scheme involved split polarization stages [Evans, 1949] with a smaller number of polarizers than in the classical scheme. The filters had a narrow, around $0.5 \AA$, passband and high contrast (the relation between transmittance in false peaks and main peak) due to the additional repeated polarization stage introduced into the scheme [Öhman, 1958]. Solar telescopes produced fairly good images with $\mathrm{H} \alpha$ filters. All these filters had almost identical life history: polarizers faded owing to inadequate protection of filter optical stack from solar radiation.

Of particular interest is the history of the first B. Halle filter, received by SibIZMIR in 1961, that was employed for year-round observation performed at the Sayan Solar Observatory. In heavy frost, the diesel power station failed, the filter was not put away into a warm place in time, and there emerged defects in the optical stack. Optical enterprises could not repair the filter. And only A.B. Gilvarg, the optician from the Institute of Crystallography, Russian Academy of Sciences, a creator of the first domestic BF [Severny, Gilvarg, 1949], helped to test the filter. It appeared that the optical stack consisting of nearly 60 elements was cemented with Canada balsam. In thermostat, at high temperature, at the risk of overheating/damaging the polarizers, the stack was disassembled and defects were found: calcite crystal plates were cracked.

With a desire to repair the unique instrument ourselves, we began to search for crystals and to gain familiarity with optical technology. We developed precision methods for processing and controlling $\mathrm{BF}$ crystal plates [Kushtal, Skomorovsky, 2002], reconstructed the out-of-operation B. Halle H $\alpha$ filter, and worked out new BFs [Aleksandrovich et al., 1975; Kushtal, Skomorovsky, 2000]. In actual fact, all B. Halle filters of the USSR solar observatories were repaired and got "second and third light" at the Optical Laboratory of our Institute. 
Into the chromospheric telescope of the Baikal Astrophysical Observatory, we put a B. Halle H $\alpha$ filter of younger, 1968, generation. Until 1980, it worked at the Horizontal Solar Telescope. Such filters are still exploited at many observatories. We utilize the BAO filter to describe the method adopted to tune the filter, present the optical scheme, main blocks, and kinematics of the filter (Figures 3, 5). Features of certain elements and stages of the optical stack of this filter as well as schemes of filters known at that time are described in [Skomorovsky, Ioffe, 1980].

In B. Halle filters of this generation, optical stack elements, except for film polarizers cemented between glasses, were assembled by immersion. This facilitated disassembly of the optical stack. Over the 35-year period of the B. Halle filter operation at the chromospheric telescope, BF has been repaired at BAO several times: we removed the blocking of the line-shifter mechanism, changed the prefilter fading owing to the heating in a beam as well as the external polarizer and polarizers inside the optical stack, which gradually faded owing to solar radiation. We established that immersion by which the crystal elements were assembled could contact with the cement layer of old or newly installed film polarizers. It gradually penetrated along the perimeter between protective glasses, producing cement defects and turbidity in the polarizers. The complete disassembly of the optical stack to replace the polarizers necessitated the production of new quarter-wave phase plates for the line-shifter because they had been made not from quartz but from crystalline mica (3, 11, 41, Figure 3 ). The very soft mica plates broke down when disassembled and cleaned, and needed replacing. It was found out that the wavefront distortions discovered in the filter and corrected by a retouched plate in "first light" were caused by the deformation of thin glass plates $(2,12$, and 42 , Figure 3$)$ installed outside of the optical stack to protect the mica quarter-wave plates. We replaced these plates as well as calcite plates damaged by severe overheating of the filter owing to the out-of-order thermoregulator.

Manufacturing BF crystal plates is a process in which during optical polishing it is necessary to ensure pinpoint accuracy of thickness of the plate, parallelism and flatness of its surface. For the Iceland spar, the production tolerance is $0.05 \mu \mathrm{m}$ for each parameter. Figure 4 shows opticians at work. Not every highlyqualified optician can make new calcite plates by hand with the required tolerance.

We have manufactured a new crystalline quartz plate $1.6 \mathrm{~mm}$ wide with edge digs within the field of view. We knew about the defect (the B. Halle company had an insufficient number of good crystals) but did not change the quartz plate because the digs were out of a solar image.

By now, all external cemented polarizers have been changed to immersion-neutral polaroid films without protective glasses. As a result, the optical stack became shorter by $20 \mathrm{~mm}$. The filter optical stack was cleaned of black dots (dust), reassembled, and "pumped" in immersion in vacuum to remove air bells (cement defects) from the field of view. The immersion-reflection index $\left(n_{\mathrm{d}}=1.578\right)$ is close to mean crystal-reflection indices for ordinary and extraordinary beams. Immersion reduces light losses due to Fresnel reflection by crystals and polarizers and compensates wavefront distortions caused by imperfect surfaces of the stack plates numbering more than 100 in the filter (Figure 3)! 


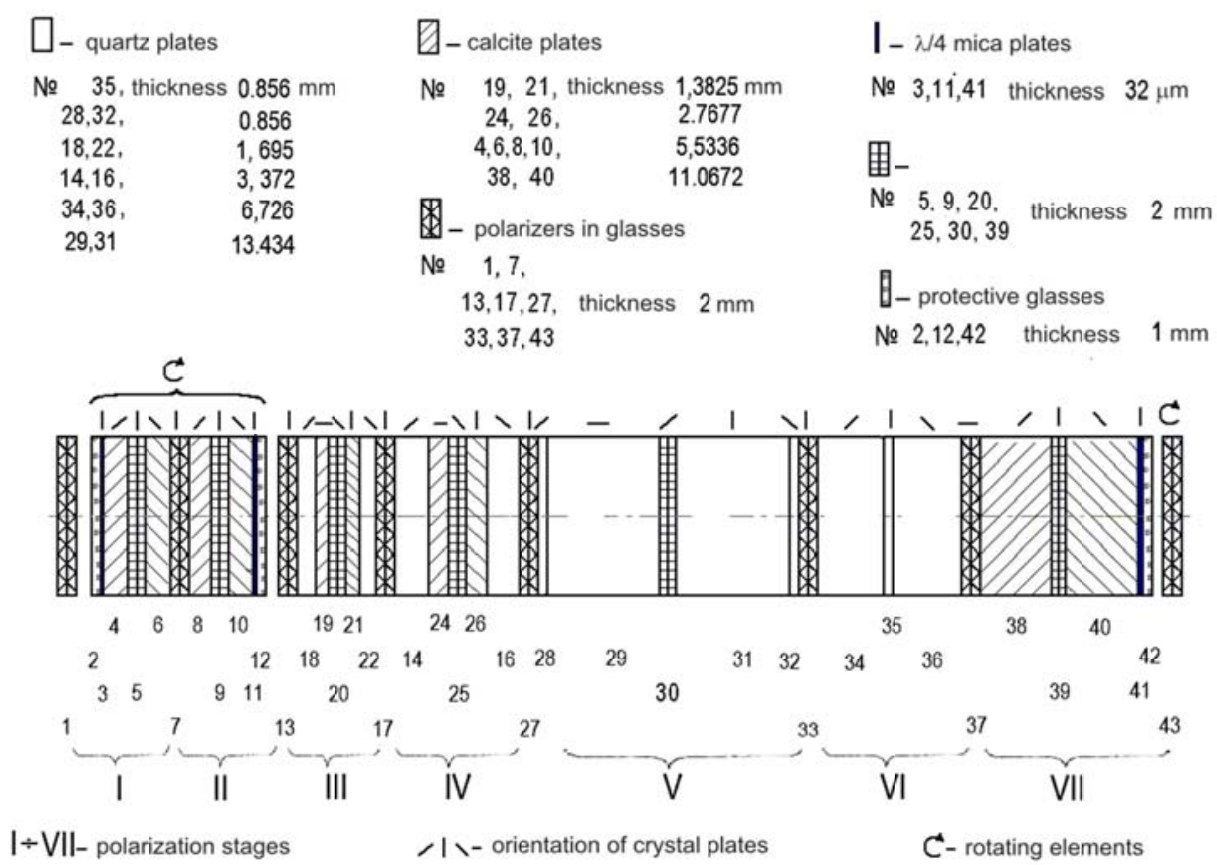

Figure 3. The optical scheme of the B. Halle H $\alpha$-filter crystal stack. Thicknesses of polarization split elements (III-IV stages) are not multiples of 2 because they contain half-wave and quarter-wave additions. Stages I-VII comprise polarizers and plates between them

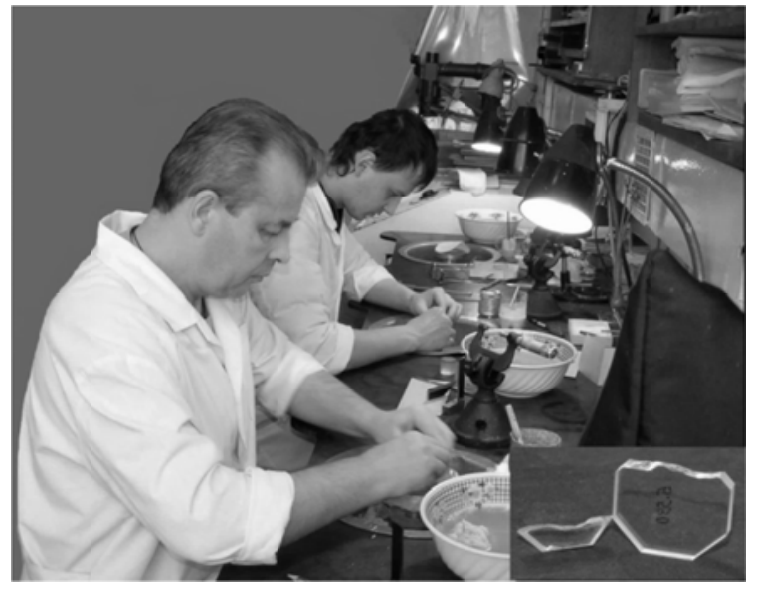

Figure 4. Opticians Khimich V.A. and Ivanov A.A. in the course of making plates for BF. In the right bottom corner is a plate damaged by overheating in BF. The wavy line of rupture indicates the nonuniform heating (thermal shock)

This filter has eleven classical BF polarizer - crystal plate - polarizer blocks combined into seven polarization stages four of which are split to reduce the number of polarizers. Three stages, I, II, VII (Figure 3), made from Iceland spar, are wide-angle, Lio I type, regular. Four stages, III, IV, V, VI with calcite and quartz plates are split, wide-angle (except for VI), Evans type. The polarization stages are located in two bushings (Figure 5). A short bushing features only two polarization stages I and II (see Figure 3). Stage II forms a $1 \AA$ passband; and stage I, the so-called contrast stage, acts virtually as the stage with a full width at half maximum (FWHM) of $1 \AA$. The contrast stage becomes effective if we include in the stack its external polarizer 1 (see also designations in Figure 3) on the lever in the flange "Contrast" (Figure 5) of the filter housing (the lever is moved from «Aus» to «Ein»). In the optical stacks, 

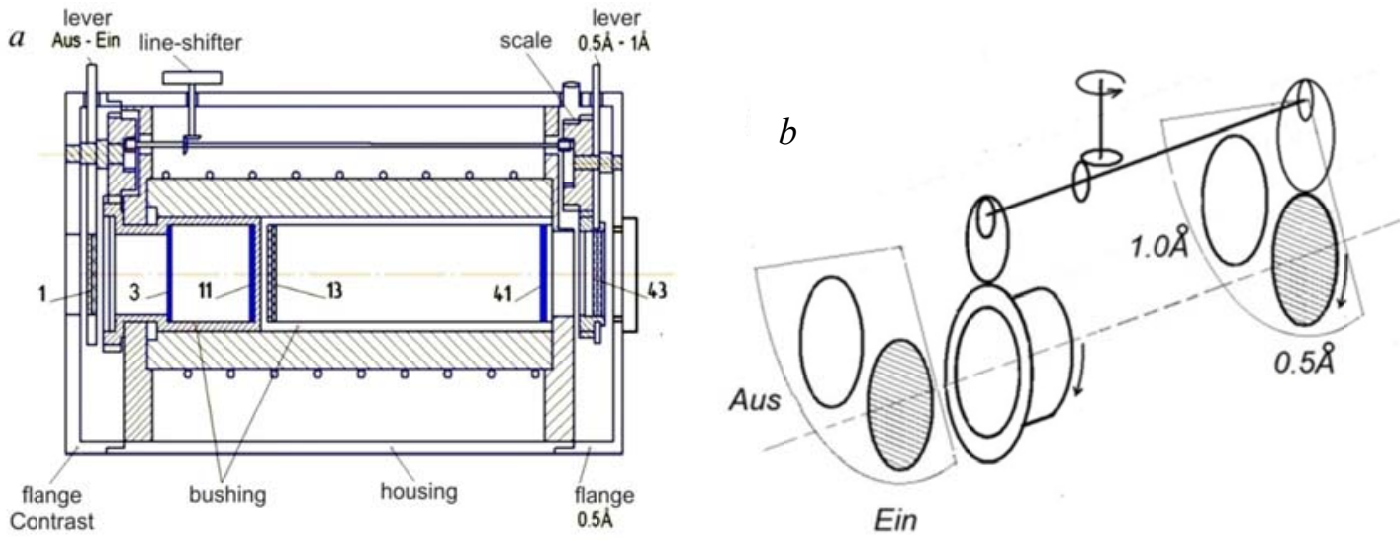

Figure 5. Barrel of the filter crystal stack (a); movable elements $(b)$

both ends of the short bushing have quarter-wave plates 3 and 11; therefore we can shift the passband of both the stages by rotating the bushing around fixed external polarizers.

A long, fixed bushing consists of five (III-VII) polarization stages. On the side of the short bushing, it has polarizer 13 of stage III. On the opposite side is quarter-wave plate 41 of stage VII with an FWHM of $0.5 \AA$. This stage becomes effective and tunable with external polarizer 43 located on the lever of flange $0.5 \AA$. The lever is moved from «1 $\AA$ » to « $0.5 \AA$ » position. The passband in all the three tunable filter stages is shifted from the general bushing (line-shifter, Figure 5) through conventional transmissions by rotating the short bushing around fixed polarizers 1, 13 and by synchronous rotation of external polarizer 43 around quarter-wave plate 41 of the fixed stage with the $0.5 \AA$ FWHM in the long bushing. The short bushing and external polarizer 43 are conventionally hooked with the 1:2 transmission ratio.

\section{ADJUSTMENT OF THE BF PASSBAND}

The goal of the adjustment is to match passbands of tunable stages, with the passband of untunable stages, and with the $\mathrm{H} \alpha$ line at operating temperature. An inaccurate matching of the passbands causes a decrease in transmission and an increase in secondary peaks.

During adjustment at the spectrograph, we should reduce the effect of such a factor as light polarization in the spectrograph and illumination system lenses upon the accuracy of stage matching. The filter flange with the contrast and $1 \AA$ tunable stages should be directed to the lamp; and the flange with the $0.5 \AA$ stage with the turned-out external polarizer, to the spectrograph slit. First, we match the passband of the $1 \AA$ stage with the filter passband by rotating the short bushing with its turned-out polarizer. Yet on the spectrograph slit we put a depolarizer to neutralize the disturbing light modulation from the $0.5 \AA$ stage. Then, we adjust the contrast and $0.5 \AA$ stages for maximum transmission by tuning their own operating polarizers. It is very useful to employ first the additional polarizers to adjust transmission of these stages, using not maximum but minimum transmission and symmetry of secondary peaks. This adjustment is more accurate. Then, the working polarizers are simply set to crossed position with the additional ones. After having matched the stages, we correct the operating temperature of the filter to accurately adjust the passband to the hydrogen line. 


\section{PROFILES OF THE BF AND INTERFERENCE PREFILTER PASSBANDS}

Spectral characteristics of BF were found at the laboratory autocollimating spectrograph ( $F=6 \mathrm{~m}$, the aperture ratio $1: 30$, the grating $200 \times 300 \mathrm{~mm}, 600$ groove $/ \mathrm{mm}$ ). The spectrograph and its equipment are detailed in [Skomorovsky et al., 2015]. The filter was set in the parallel beam; spectra were registered by the CCD linear array MORS. Figure 6 illustrates spectra of the passband at $0, \pm 0.25, \pm 0.50$, and $\pm 0.75 \AA$ from the $\mathrm{H} \alpha$ line center. Further shift of the passband leads to a considerable increase in a secondary peak because other filter stages are untunable. Within the $\pm 0.5 \AA$ shift, false peaks are low, and the passband has a high contrast.

In 1989 (before the current repair), for preliminary monochromatization in BF we installed an interference MDM heat filter and a narrowband dielectric interference filter. Coatings of these filters were in vacuum therefore they were well protected from moisture [Proshin, Aleksandrovich, 1982], but silver layers were damaged by solar radiation. In the photograph (Figure 7, top right), this can be seen in the central part of the MDM filter (a reflection glare). The MDM-filter transmittance in the center became two times less than at the edge. Both the filters have a good wavefront (for transmission), but, because they were sealed in vacuum, their glasses were deformed under atmospheric pressure. The filters must not be cemented together - they should be installed into the optical stack with an air gap between them to leave the wavefront unaltered. Re-reflections in the air gap can cause reflection glares and false suns in an image owing to obliquity of BF. The sensitivity area of the CCD camera extends farther than $1.1 \mu \mathrm{m}$, therefore we supplemented the tandem MDM-filter+IF with a color glass light filter SZS-25. Figure 8 shows the passband of the IF tandem in the main peak in 1989 (1), its absorption in a broad spectral region (2), and absorption of the SZS-25 filter (3) that completely eliminated secondary peaks at a distance of more than $0.9 \mu \mathrm{m}$, thus increasing the optical density of the tandem from 2 to 6 . The total transmittance of the assembly is not given in the figure.
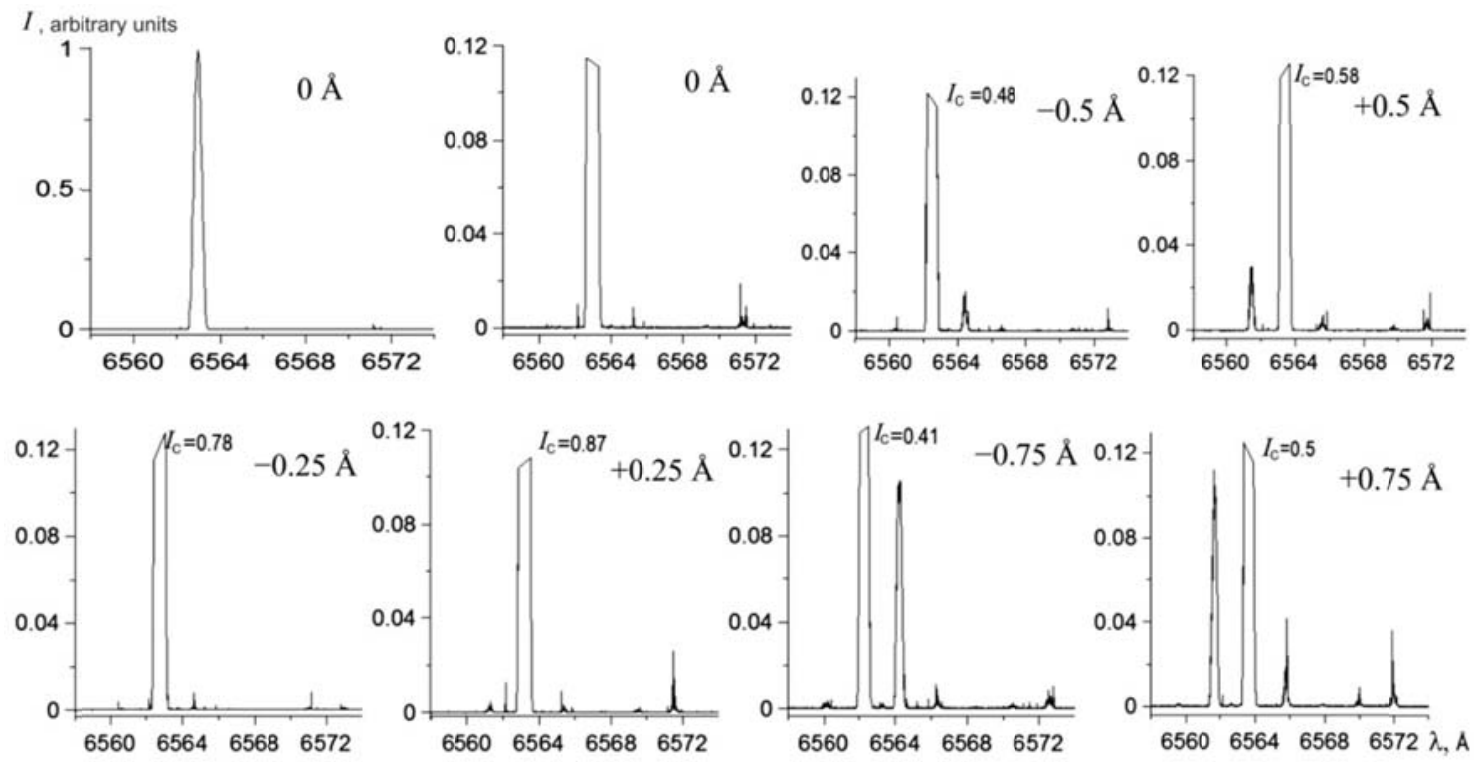

Figure 6. BF passbands for $0, \pm 0.25, \pm 0.5$, and $\pm 0.75 \AA$ positions from the $\mathrm{H} \alpha$ line center. They are normalized relative to the passband in zero position. Filter transmittance at zero position of the line-shifter. Profiles for all the positions are scaled up to distinguish the false peaks. As the passband shifts, the intensity $I_{\mathrm{c}}$ of the main peak reduces. The FWHM decreases from $0.43 \AA$ at $0.25 \AA$ to $0.39 \AA$ at $0.75 \AA$ 

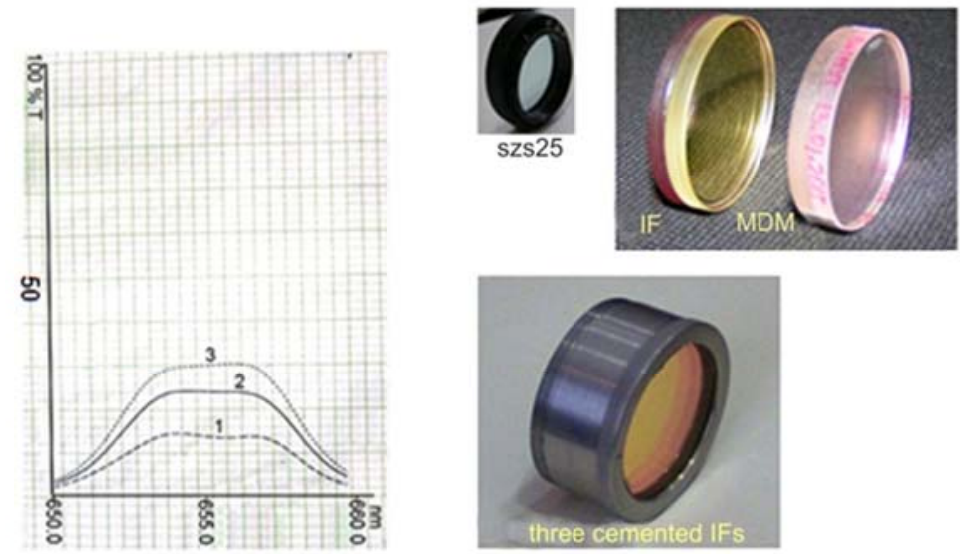

Figure 7. On the right are prefilters of 1989 (at the top) and 2015 (at the bottom). On the left is the transmittance of the MDM filter in the center (1) and on edges $(2,3)$

The new prefilter developed in 2015 is comprised of three dielectric filters: a narrowband contrast one with an FWHM of $\sim 30 \AA$, and two filters cutting up to $1.4 \mu \mathrm{m}$. Nineteen-layer cut filters are designed

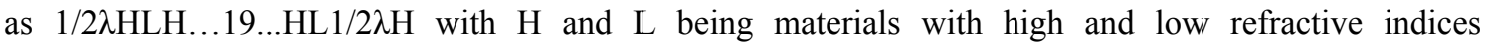
respectively. Yet external coatings are half-wave, made from $\mathrm{ZnSe}$ with a high reflective index; and internal coatings are quarter-wave, applied alternatively by materials with high $(\mathrm{ZnSe})$ and low $\left(\mathrm{Na}_{3} \mathrm{AlF}_{6}\right)$ refractive indices. The multilayer filter coatings are sealed by optical cement. All the three filters are cemented together and set in the barrel (the bottom photograph on the left of Figure 7). Figure 8 illustrates the passband of the narrowband IF (4), the IF optical density with one cut filter (5), and the optical density of the IF assembly with two cut filters (6).

At $22{ }^{\circ} \mathrm{C}$, the narrowband filter is centered on $\lambda 6581 \AA$, the transmittance is $69 \%$; and in the $\mathrm{H} \alpha$ line, the transmittance is $\sim 30 \%$. This filter with the $30 \AA$ FWHM well suppresses secondary peaks of BF. Although the prefilter is not centered on the hydrogen line, there is enough light, transmitted by the IF+BF system, to capture images by the new camera with exposure time of several milliseconds.

\section{REIMAGING LENS}

The primary solar image $20 \mathrm{~mm}$ in diameter is produced by the teleobjective and field lens approximately in the middle of BF. The new four-lens objective (Figure 9) transfers the image to the Hamamatsu C9300-124 digital camera (Japan). The matrix size is $24 \times 36 \mathrm{~mm}$; the image diameter, $18 \mathrm{~mm}$. The objective also corrects aberrations of prior optics. Since the laboratory had a limited set of radius operating test glasses, the objective was calculated as a two-component system. Each of the components included two lenses (crown, flint) with the use of only two nominals of radii of curvature.

When the lenses were being treated, the interference control of the shape of their surfaces was made with the operating test glasses; and the control of centering, by measuring thickness of the lenses along the edge on a rotating table (Figure 10) with the aid of an indicator. 


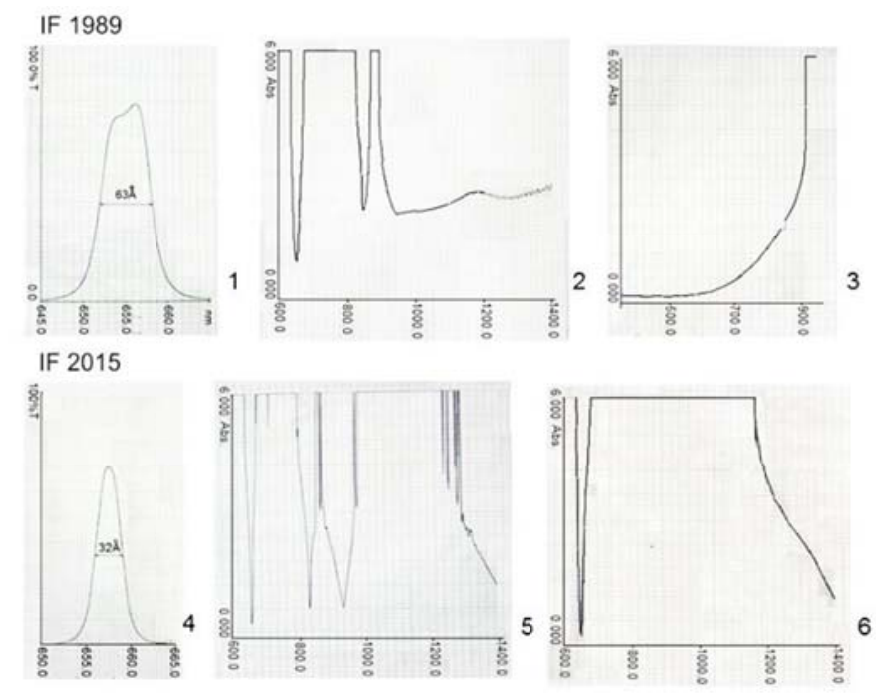

Figure 8. Spectra of prefilter transmissions obtained by the Hitachi U-3400 spectrophotometer. Along the X-axis is the wavelength in nanometers; along the Y-axis, the transmittance in $\%(1,4)$, and the optical density in absorption $(2,3,5,6)$

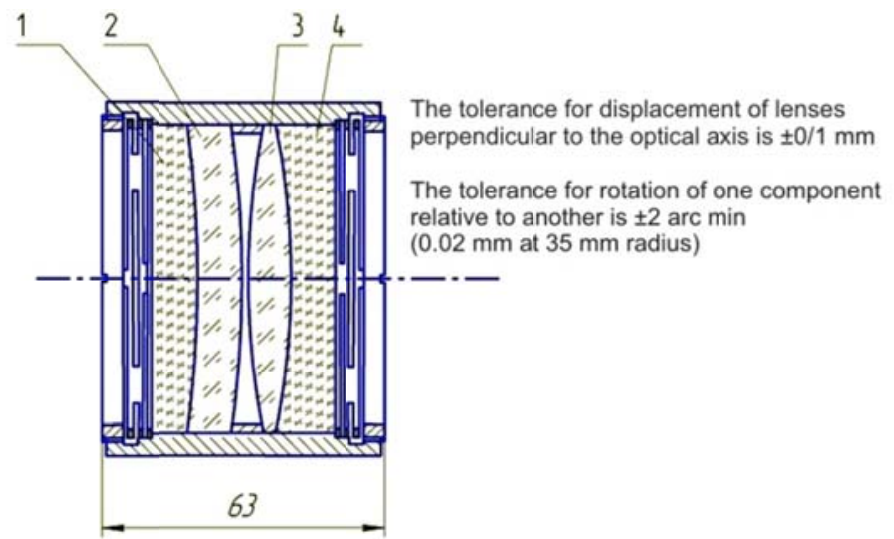

Figure 9. Reimaging lens $(F=230 \mathrm{~mm}, D=70 \mathrm{~mm})$ in the barrel: 1,3 are positive lenses; 2 is a meniscus; 4 is a magnetic-base negative lens

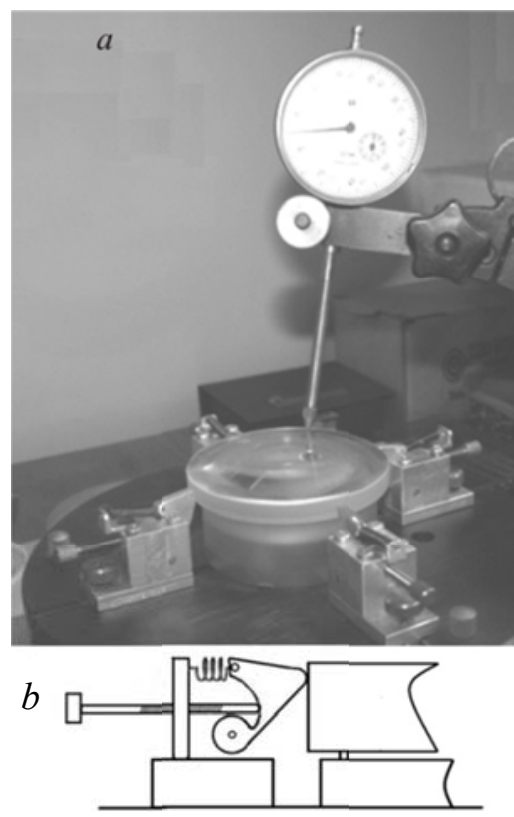

Figure 10. Table for measuring wedging of lenses $(a)$; pressing scheme $(b)$ 
Before measuring lens thickness, we should press the lens to the support of the rotating table and set it (with the same indicator) in such a way as to prevent its eccentricity when the table rotates. A shortcoming of most well-known table designs is that the radial pusher lever not only shifts but also can slightly raise a lens, thus introducing an error into the control of its thickness along the lens edge. To improve the control accuracy, we worked out a new pendulous design for the pusher. The pusher side bearing up against the edge of the lens is beveled and rounded; the reverse side is made in the form of evolvent along which an adjusting screw during rotation slides and moves the pusher. The pusher itself can sway on a spindle, located below the adjusting screw. When rotating, the screw presses the pusher always at right angles to the evolvent, and the pusher, displacing the lens lengthwise, simultaneously presses it to the support of the rotating table.

Each component of the reimaging lens is assembled by immersion with a refractive index $n_{\mathrm{d}}=1.578$. The interference quality control of the lens was run with an unequal-arm interferometer in the autocollimation scheme (Figure 11) in the best image plane.

Figure 12 shows a calculated wave difference of the beam path in the four-lens reimaging lens for an axial point $(a)$ and a wavefront interferogram in the double beam path through the lens $(b)$. Wave aberrations of the reimaging lens are close to estimated ones and range within $0.1 \lambda$.

\section{ASSEMBLY AND ADJUSTMENT OF THE TELEOBJECTIVE IN THE STANDARD BARREL}

Over the 35-year period of operation, the teleobjective became dirty and needed complete disassembly, cleaning, and readjustment.

The standard barrel of the teleobjective is a tube about $400 \mathrm{~mm}$ long (Figures 13, 14). It features adjustable tilt of the negative lens on the ball support of the back tube flange and adjustable lengthwise displacement of this lens relative to the positive lens, fixed motionlessly on the front tube flange. There is no mechanism for lens displacement perpendicular to the optical axis because exact centering of lens barrels in the tube of the teleobjective and lenses themselves is specified by their design. The installation of puller bolts to the back flange of the teleobjective tube has proved useful in practice. They can help to remove the blocking of the negative lens barrel with the lens tilt being adjusted on the ball support. In the tube sidewall there is a port through which we can put a baffler to eliminate secondary reflection glares during lens adjustment.

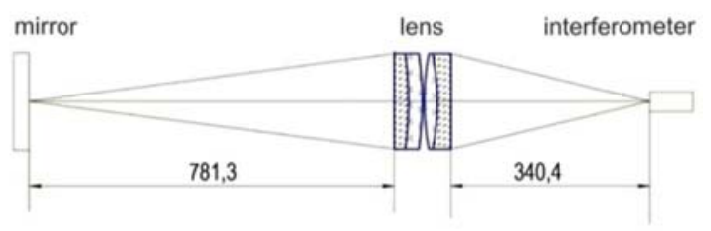

Figure 11. Control scheme in the best image plane 


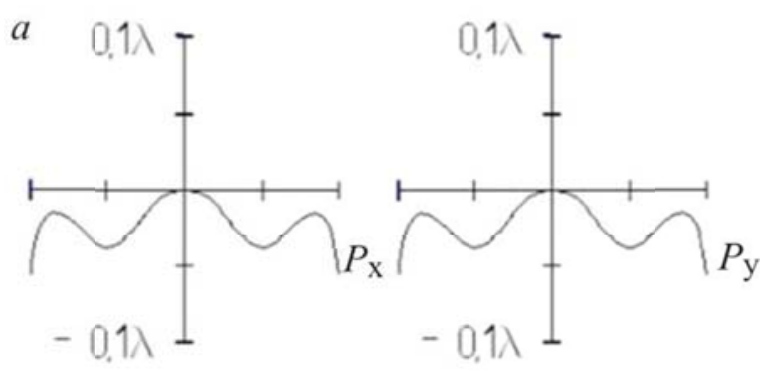

b

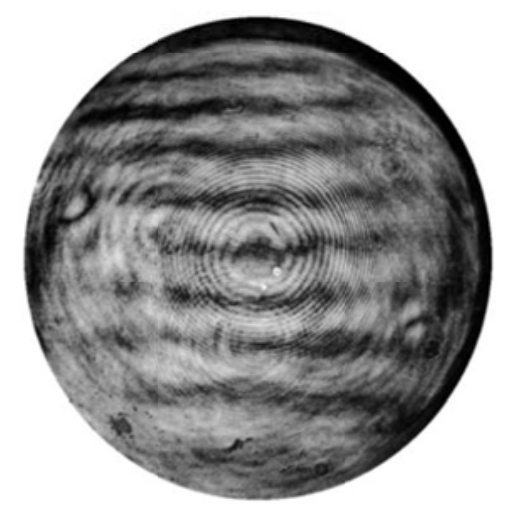

Figure 12. Wavefront of the lens for an axial point: $a$ is a computed wavefront, $P_{\mathrm{x}}, P_{\mathrm{y}}$ are radii of the entrance pupil in meridional and sagittal planes; $b$ is a wavefront interferogram (double path), $\lambda 6328 \AA$

The teleobjective lenses are installed into their barrels 1, 4 (Figure 13) on three side spacers placed at an angle of $120^{\circ}$. To the same barrels during the lens adjustment, we fasten diaphragms 7, 9 (FIgure 13) with apertures $1-2 \mathrm{~mm}$.

These apertures are used to center a beam of the laser, installed together with screen 5 , on the side of the positive lens at a distance of $\sim 1 \mathrm{~m}$ (Figures 13,15). The beam traces the position of the geometrical axis of the teleobjective tube. The positive lens is nonadjustable. It is set perpendicular to the optical axis by tilting and transversely displacing the whole tube by optical bench mechanisms.

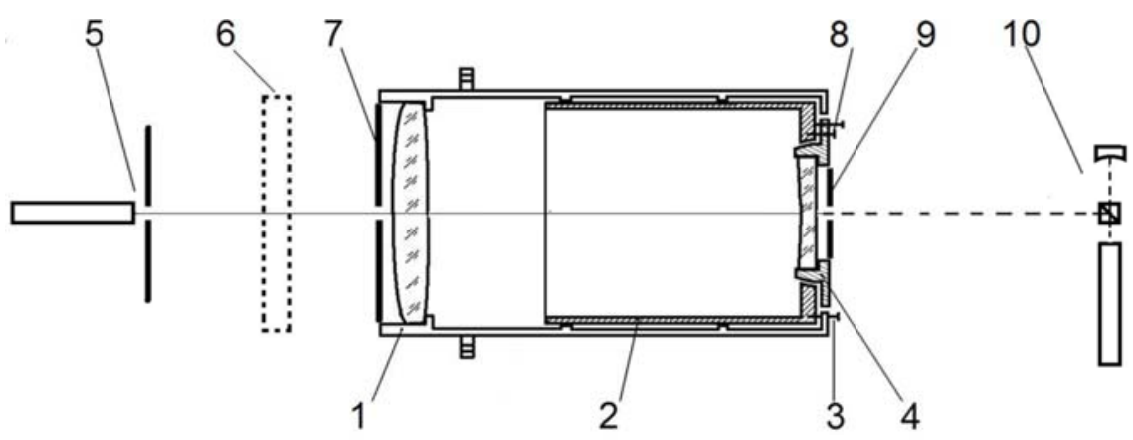

Figure 13. Adjustment and control of the teleobjective in the barrel: 1 is the external tube (positive lens barrel); 2 is the internal tube with a ball support; 3 is the adjustment of inter-lens distance; 4 is the negative lens barrel; 5 is the laser with the screen; 6 is the reference flat mirror; 7 is a diaphragm; 8 are the adjustments of the negative-lens tilt; 9 is a diaphragm; 10 is the unequal-arm interferometer 


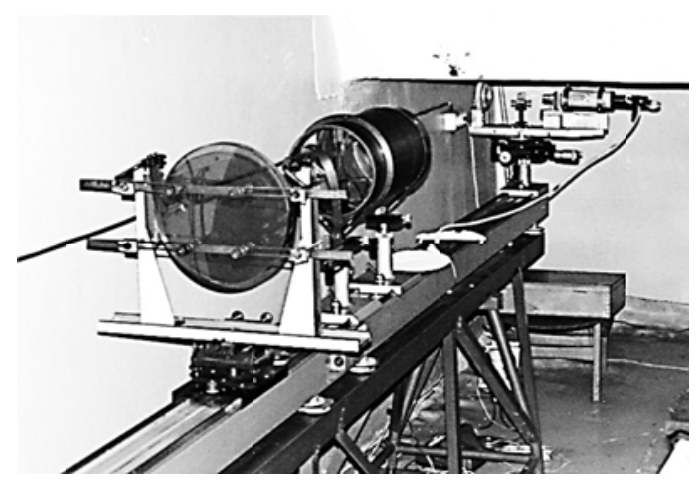

Figure 14. Device with the flat etalon and interferometer to control the wavefront of the teleobjective

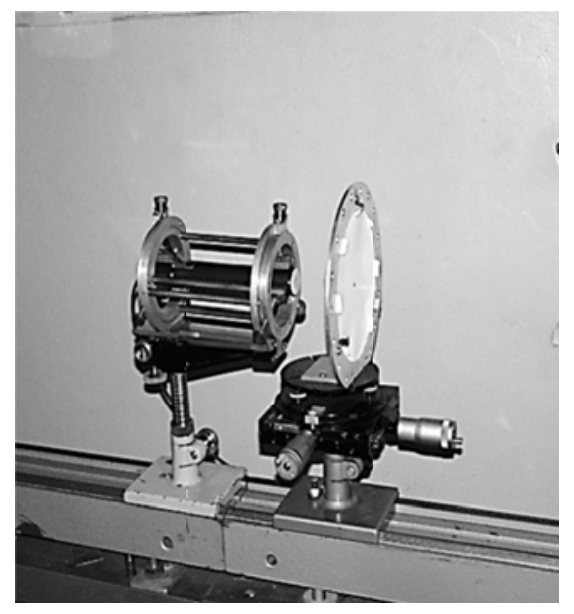

Figure 15. The laser with the screen
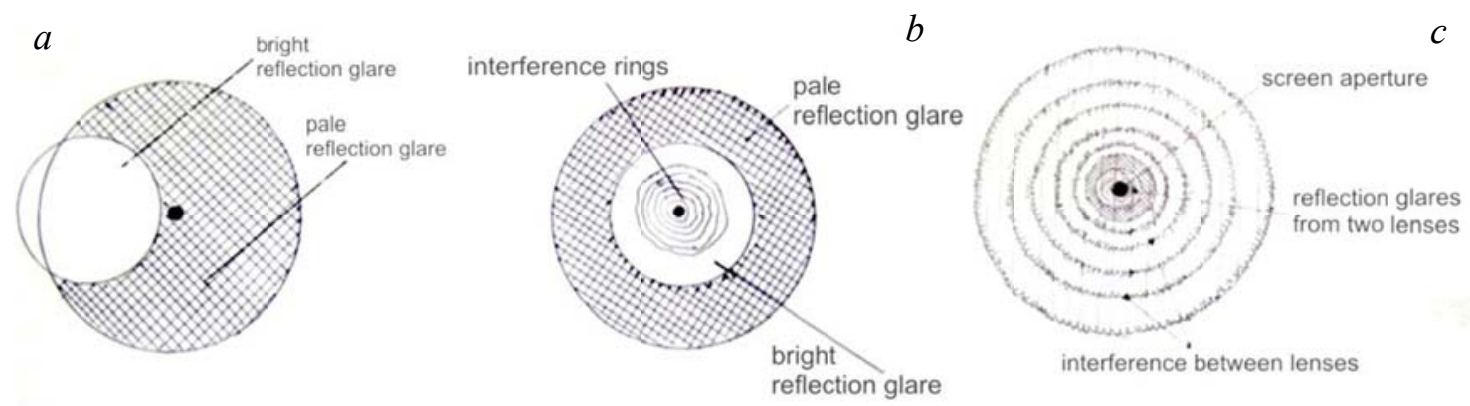

Figure 16. Appearance of an interference image during matching of reflection glares. Reflection glares only from the positive lens are separated $(a)$, coincide $(b)$. Reflection glares from four surfaces of the two lenses coincide $(c)$

The control is implemented on the screen, using reflection glares reflected from the lens surface. A reflection glare from its front convex surface is blurred; that from its back concave surface, focused (Figure 16,a). As reflection glares approach each other, we observe an interference ring image that is centered at the screen aperture by tilting the lens (tube) (Figure 16, $b$ ). Reflection glares from the negative lens can be temporarily blocked with a baffler lest they impede. Nevertheless, we should control beam propagation through apertures of the diaphragms of both the lenses and, if necessity, correct the laser's position.

After adjustment with reflection glares from the positive lens, we firmly fix the teleobjective tube on the optical bench. Reflection glares from the negative lens are also centered at the screen aperture by adjusting the tilt of the barrel on the ball support before the interference image appears within this lens and 
between lenses. When reflection glares from the lenses are situated near the screen aperture, they are precisely centered at the aperture by tilting the lenses; and interference images are displaced and centered by parallel displacement of the lenses. If centers of three images cannot be matched by changing the negativelens tilt, it is necessary to displace the lens in the barrel toward matching of the images by changing spacers. The screen (Figure 16,c) exhibits three systems of Newton interference rings: two from the lenses and one, wider, from surfaces of the air gap. The latter reacts to vibrations in a building and air turbulence in it. With required tolerances for centering of the lens, the admissible spacing between image centers is no more than $0.5 \mathrm{~mm}$ at a distance of $2 \mathrm{~m}$ from the lens to the screen [Klevtsov, 1987].

\section{STUDYING THE WAVEFRONT OF THE TELEOBJECTIVE}

In focus of the teleobjective, we set unequal-arm interferometer 10 (see Figures 13, 14) centered at the same apertures of the diaphragms on barrels of the teleobjective lenses; and on the side of the positive lens, we install reference flat mirror 6 to control the wavefront in the autocollimation scheme. Note that the teleobjective was adjusted using special laser 5 instead of interferometer laser 10 (Figure 13) because in the beam splitting cube of the interferometer there occurred additional reflection glares impeding the adjustment.

Wavefront interferograms in the double beam path were obtained for a point on the axis of the teleobjective with a distance of $345.5 \mathrm{~mm}$ between the lenses. With this distance, the teleobjective - field lens system produces an intermediate solar image in the middle of the BF optical stack. Figure 17 shows interferograms obtained in 2015 before disassembly $(b)$ and after cleaning and assembly $(c)$. For comparison, we give the previously presented wavefront interferogram (Figure 2) of the lens after the first assembly in 1980 (Figure 17,a). Below are calculated wavefront profiles for axial points and at a distance of $16 \operatorname{arcmin}(d)$.

Over the 35-year period of the telescope operation, the adjustment and wavefront of the teleobjective have changed little, if at all. The newly adjusted lens shows the wavefront not worse than before disassembly, and even closer to the calculated value. Differences between interferograms can be caused by airflows in the building and by temperature gradients on the lenses that appear despite air blowing of the control zone.

\section{ADJUSTMENT OF THE WHOLE OPTICAL SYSTEM OF THE CHROMOSPHERIC TELESCOPE}

On the optical bench, we set the telescope tube without optics (Figure 18) assembled from the following blocks: teleobjective flange 1, declination box 2, filter 3, and reimaging lens 4 with the camera. On the side of the reimaging lens we fix the unequal-arm interferometer with the screen. The camera is not shown in the figure. In the front flange of the telescope and in the mount of the reimaging lens barrel, we set diaphragms D1, D2 with a central aperture 1-2 $\mathrm{mm}$. These apertures are reference for tracing of the future optical axis of the telescope and for installation of teleobjective optics, field lens, BF, reimaging lens, and CCD camera. 

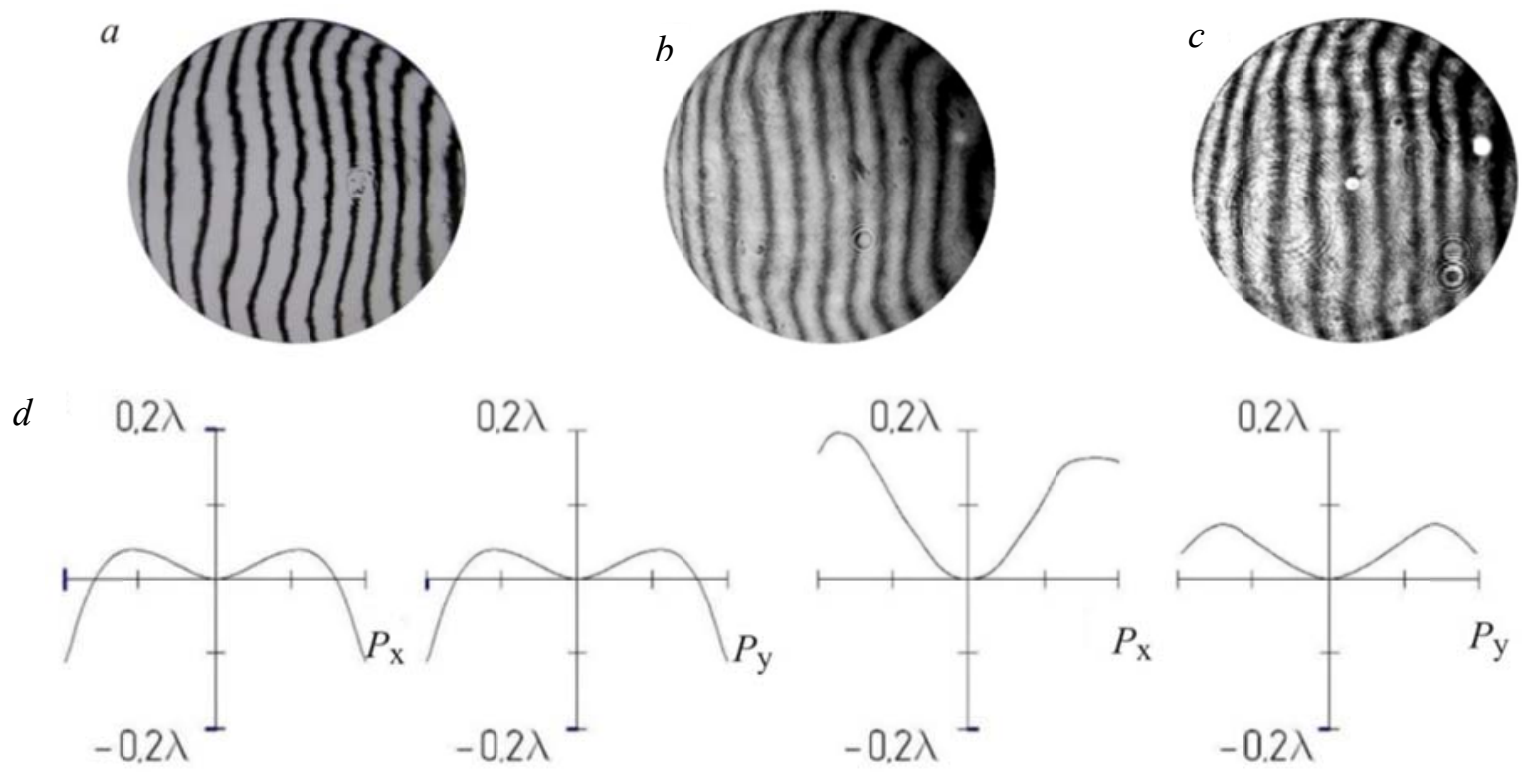

Figure 17. Wavefront interferograms of the teleobjective: $a$ is 1980, the first assembly [Klevtsov, 1987]; $b$ is 2015 before the disassembly; $c$ is the assembly on May 5, 2015; $d$ is the calculated wavefront
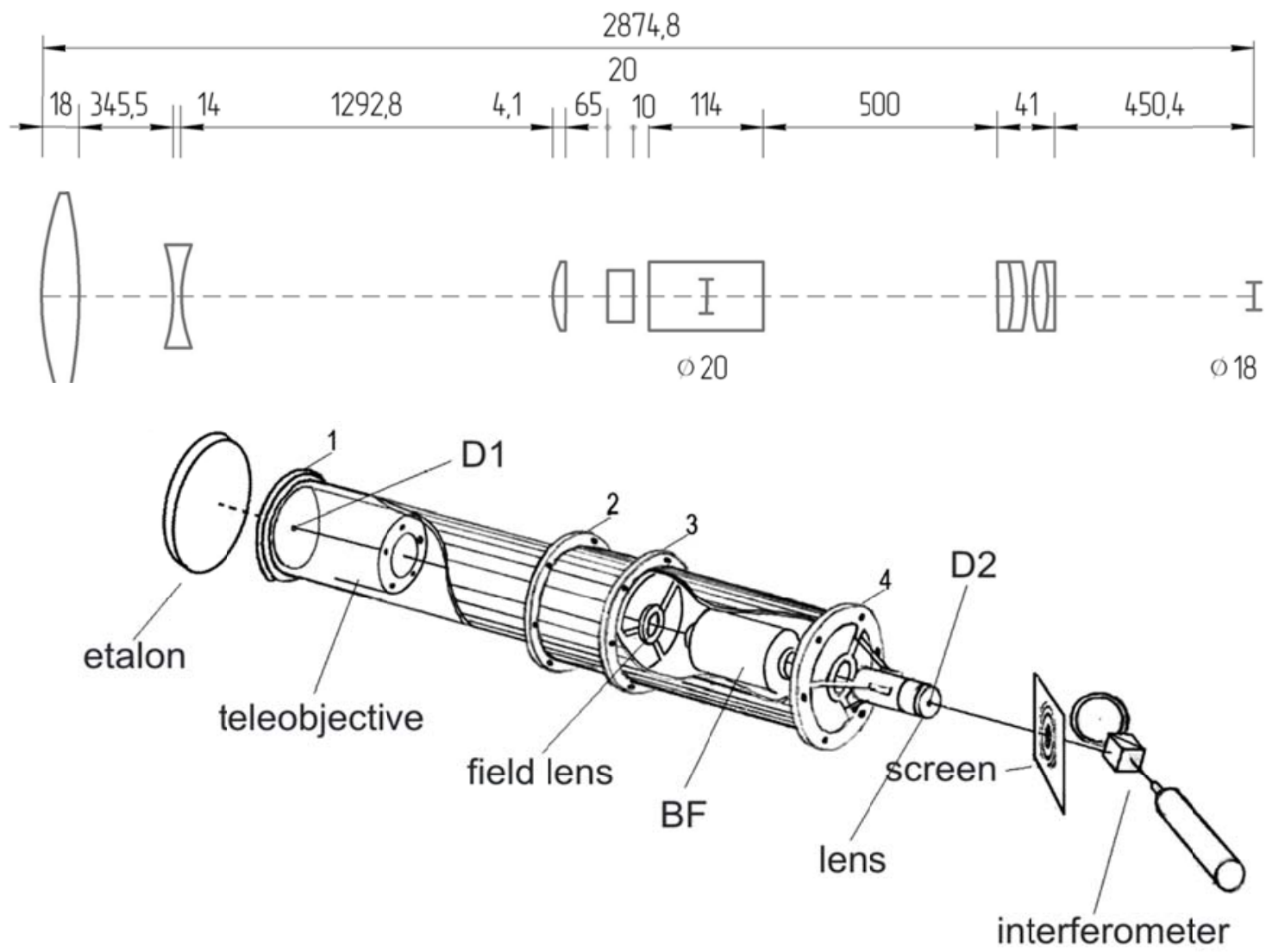

Figure 18. Adjustment and control scheme for the telescope The numbers at the top are distances between optical elements, $\mathrm{mm}$ 
The beam splitting cube of the interferometer is changed to a mirror prism for the adjustment period to get rid of secondary reflection glares. By moving the interferometer, we direct a laser beam from the prism through the aperture in the screen to the telescope and match it with the telescope axis through reference apertures of the diaphragms.

The optical elements are set and adjusted according to their position in the optical scheme, starting with the teleobjective.

The teleobjective. The teleobjective, assembled and adjusted in its barrel, is fixed in the front flange of the telescope. By tilting the whole barrel, we adjust an interference image of reflection glares, which reflected from surfaces of the teleobjective lenses, to the center of the interferometer screen.

The field lens is installed into its barrel. In this case it appeared that the optical center of the field lens did not coincide with the center of the barrel. We had to turn the mount of the barrel and shift the field lens along with the barrel by $1 \mathrm{~mm}$ to make the reflection glares reflected from its surface be within the screen center.

$B F$. If the filter is set correctly, a laser beam should come to the center of the protective glass of the BF optical stack and be reflected to the interferometer screen center. We gave surfaces of protective glasses in the filter an anti-reflection coating for the red spectral region. To enhance brightness of the reflected laser beam, we screwed a glass in barrel (mirror or cut filter) into a window flange of the filter. The surface of the glass had been made parallel to the BF protective glass. Notice that the surface of the entrance IF should also be set parallel to the entrance (exit) window of the crystal stack. BF is brought to the optical axis and to autocollimation by longitudinal and inclined movements of a matching site. After the adjustment, BF is taken out from the matching site because its passband does not transmit emission in the $6328 \AA$ line. To control the wavefront of the telescope in this line, we replace BF with a simulator made from a transparent glass.

The reimaging lens. The mount of the lens barrel with the installed base diaphragm D2 is preliminarily set perpendicular to a laser beam, using autocollimation from an auxiliary mirror. The objective lenses themselves and the lens barrel were well centered and after installation of the barrel to the mount fell in the screen center.

The landing flange of the CCD camera (not shown in the figure) was adjusted to the axis of the telescope, using a laser beam.

\section{WAVEFRONT OF OPTICS OF THE CHROMOSPHERIC TELESCOPE AND “NEW LIGHT”}

Wavefront interferograms of the telescope (Figure 19) were obtained in the autocollimation scheme with the reference flat mirror. Before exposure, optics had been «kept» for several hours to equalize temperature gradients. The building where all the optical elements were located was blown by a fan. 


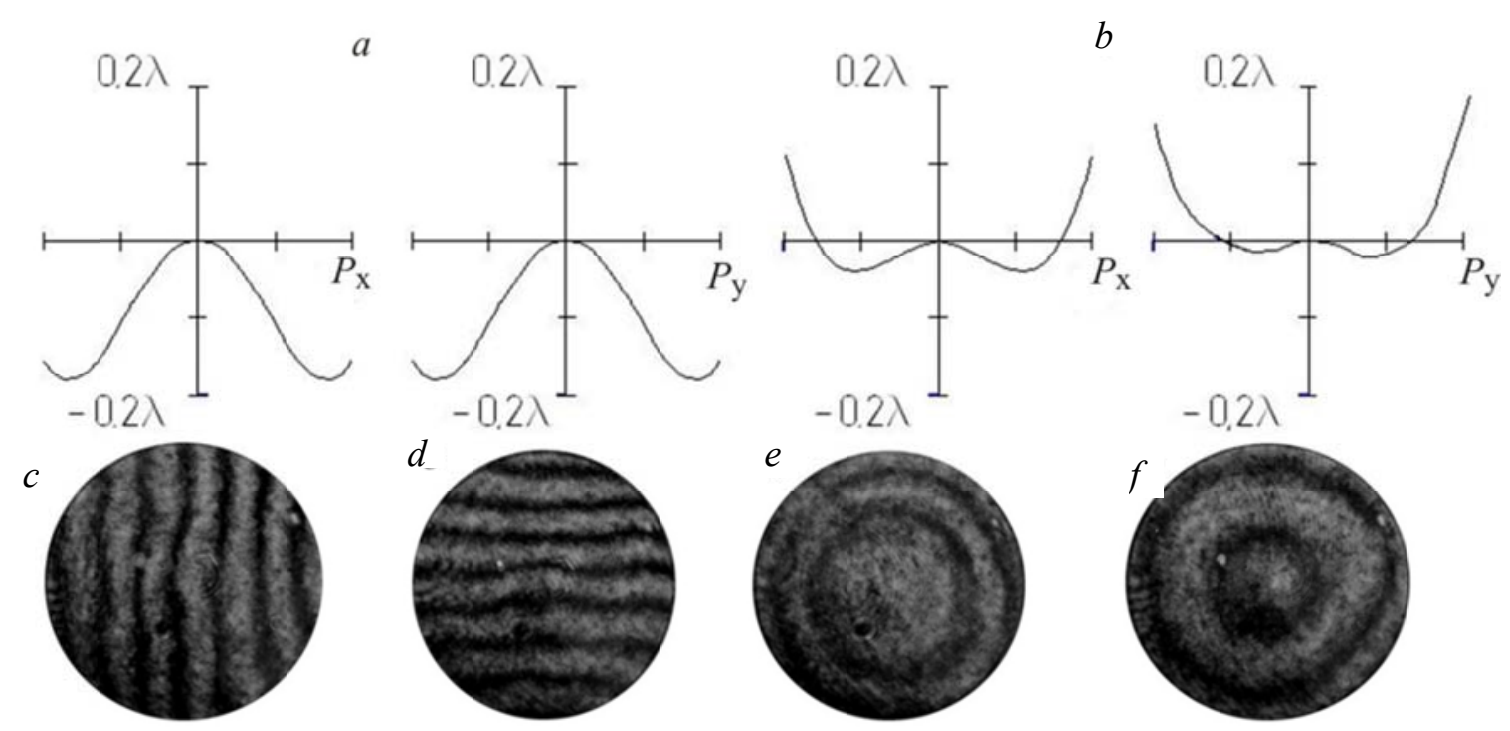

Figure 19. Wave aberrations of the H $\alpha$ telescope optics: calculated wave aberrations $(\lambda=0.6563 \mu \mathrm{m})$ for an axial point $(a)$; those at the edge of the field of view of $16 \operatorname{arcmin}(b)$; interferograms $(\lambda=0.6328 \mu \mathrm{m})$ in the double beam path for an axial point, focal images $(c, d)$; a postfocal image $(e)$; a prefocal image $(f)$

The interferograms show that the quality of the telescope optics ensures fulfillment of the Rayleigh criterion. Wavefront distortions of the modernized BF, which were measured using separate blocks in the Twyman interferometer, are also within tolerable limits. Below is given a full disk chromospheric image and scaled-up fragments of the images captured in the $\mathrm{H} \alpha$ line center and wing with an aperture of $120 \mathrm{~mm}$ and exposure time of $0.005 \mathrm{~s}$ in summer 2015 (Figures 20-24). The FWHM is $0.47 \AA$. Spatial resolution is $\sim 1 "$.

Given the nominal diameter of the lens of $170 \mathrm{~mm}$ and its high quality, it is possible to obtain higher resolution images under good atmospheric conditions; however, the effective diameter of the lens has been decreased because a full-disk image is taken at resolution of the telescope already determined by matrix and pixel sizes. The use of the entire aperture can lead to an increase in scattered light and to an unjustified increase in the heat load on BF.

In the telescope, by employing the full aperture of $180 \mathrm{~mm}$ and the high-resolution camera under good astroclimatic conditions, we can obtain solar images with resolution higher than 1" with image monochromaticity within $0.05 \AA$ across the field of view.

\section{CONCLUSION}

The chromospheric telescope of the Baikal Astrophysical Observatory is again ready for observations within the solar patrol program and for scientific research. We have made a new reimaging lens to take full solar disk images with the Hamamatsu C9300-124 digital camera. All the telescope optics have been cleaned and readjusted. BF has been repaired. Spectral characteristics of the prefilter and BF provide high-contrast monochromatic images. Wavefront interferograms of optical elements and the telescope as a whole show that wavefront distortions of the entire optical path are within $0.25 \lambda$. 
Over a period from 1981 to $1999,50 \mathrm{~mm}$ solar images were captured on an 80-mm film. The available archive of films has many images with spatial resolution of about 1". In 2000-2002, 50 mm images were taken by the Princeton Instruments CCD camera with a $50 \times 50 \mathrm{~mm}$ matrix detector, $2048 \times 2048$ pixels. Nowadays, $18 \mathrm{~mm}$ solar images are captured by the Hamamatsu C9300-124 CCD camera with a $36 \times 24 \mathrm{~mm}$ matrix detector, $4000 \times 2672$ pixels. There is slightly more than 1 pixel for 1 ".

It is obvious that digital recording successfully competes against film recording, but this cannot imply an overwhelming advantage of one technology over another [http://archives.ru/documents/ methodics/obzor_restore-text-archival-document.shtml]. Resolution of photomaterial depends on its photosensitivity. Proper exposure and development time of a film can provide a higher resolution image if the telescope lens is not stopped down to $120 \mathrm{~mm}$ as we are doing now to reduce useless exposure. On the other hand, even if we set its diaphragm to $120 \mathrm{~mm}$, the exposure time of the CCD camera for features on the solar disk is one order of magnitude shorter than that of film recording; it is $5 \mathrm{~ms}$ with a dynamic range of around 4000. This is essential for acquisition of tens and hundreds of images less prone to seeing effects, for selection of the best images or their post factum processing with regard to the flat field.

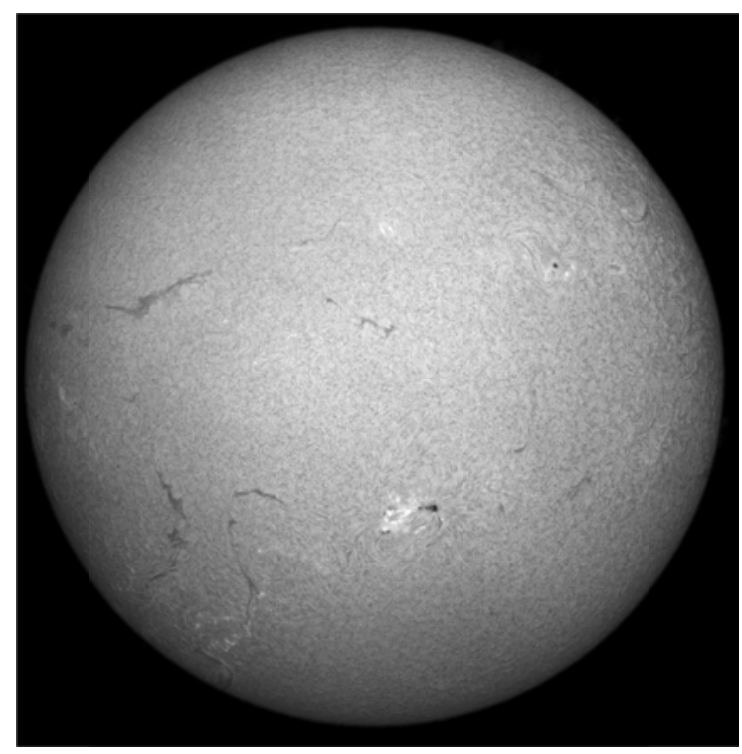

Figure 20. The chromosphere, the Ha line center, August 8, 2015, 09:01:00 LT

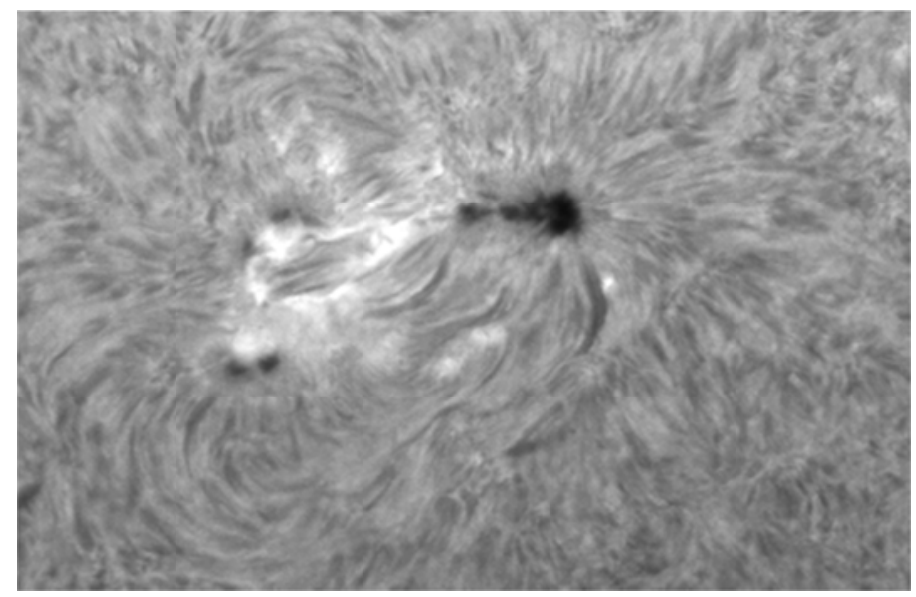

Figure 21. The chromosphere (a fragment of Figure 20), the Ha line center, August 8, 2015, 09:01:00 LT 


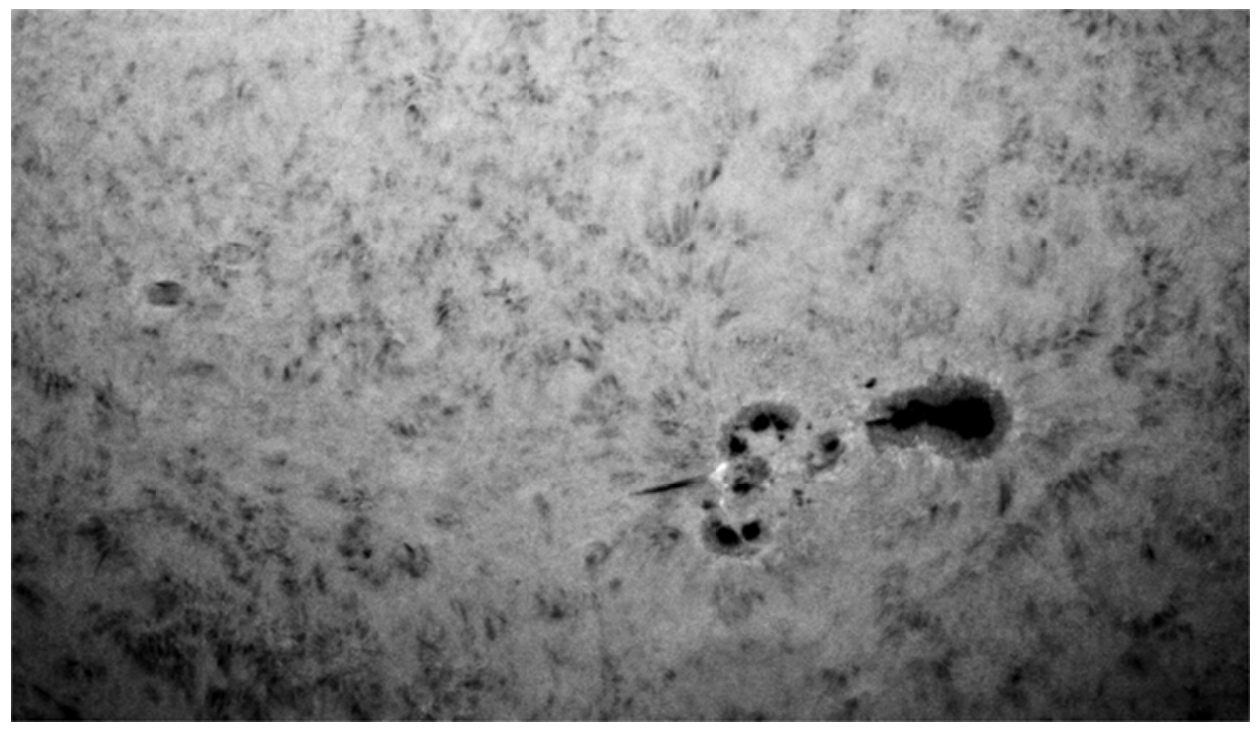

Figure 22. The chromosphere, the $+0.5 \AA$ wing, August 8, 2015, 09:06:01 LT

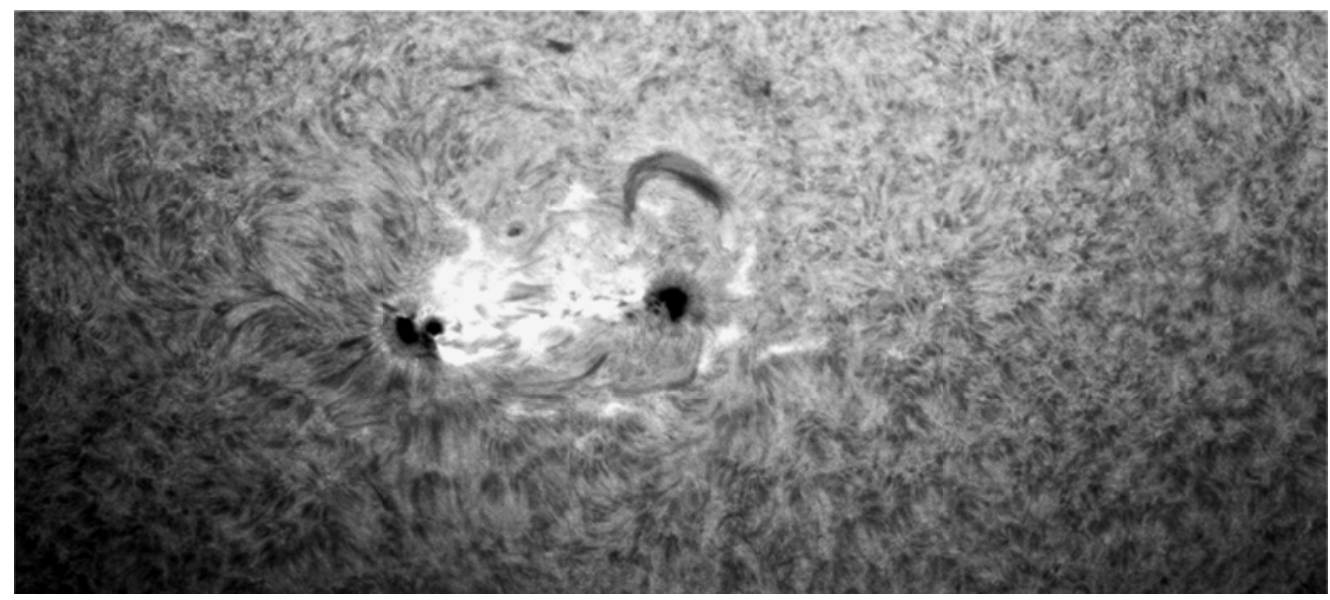

Figure 23. The chromosphere, the $+0.2 \AA$ wing, August 23, 2015, 15:21:18 LT

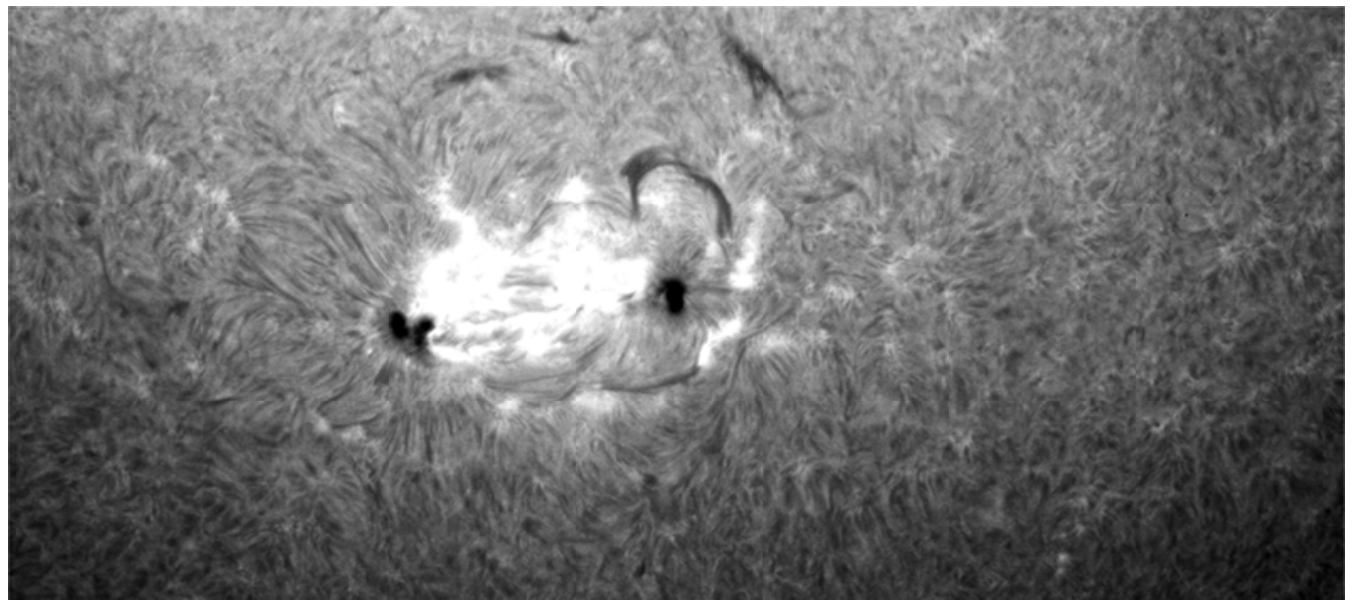

Figure 24. The chromosphere, the H $\alpha$ line center, August 23, 2015, 17:13:17 LT

This is just the digital technology that facilitates real-time registration and exchange of solar images in the worldwide network of solar observatories. Addresses of the archive of the solar patrol of the worldwide network of solar stations and real-time $\mathrm{H \alpha}$ observations are given on the website of the 
National Solar Observatory (the USA) [http://www.nso.edu/ IAU-Com12/resources, http://www.hida.kyoto-u.ac.jp/ Smart/]. You can see that in the image quality the BAO chromospheric telescope is highly competitive with telescopes of the worldwide network. Moreover, opportunity exists to further increase its resolution by means of a higher resolution matrix.

We thank C.Sc. (Phys.\&Math.) A.A. Golovko, A.A. Zhdanov not only for providing us with solar observations to illustrate operation of the telescope but also for the image quality analysis contributing to the modernization, as well as the head of the Baikal Astrophysical Observatory C.Sc. (Phys.\&Math.) A.V. Borovik - the main initiator and organizer of this great work - and the head of the machining department V.S. Fedotov who supervised the fine adjustment of the telescope units and the manufacture of technological devices for treating and controlling the telescope optics.

\section{REFERENCES}

Aleksandrovich S.V., Domyshev G.N., Korovkin A.I., Sadokhin V.P., Skomorovsky V.I. Narrow-band birefringent filter with two transmission bands. Novaya tekhnika v astronomii [New Facilities in Astronomy]. 1975, no. 5, pp. 34-39. (In Russian).

Banin V.G., Klevtsov Yu.A., Skomorovsky V.I., Trifonov V.D. Ha-cinematograph of SibIZMIR. Solnechnye dannye [Solar Data]. 1982, no. 1, pp. 90-94. (In Russian).

Banin V.G., Klevtsov Yu.A., Skomorovsky V.I., Trifonov V.D. Khromosfernyi teleskop [Chromospheric Telescope]. Patent USSR no. 1018092, 1983a. (In Russian).

Banin V.G., Borovik A.V., Yazev S.A., Big solar flares of May 13 and May 16 1981. Issledovaniya po geomagnetizmu, aeronomii i fizike Solntsa [Research on Geomagnetism, Aeronomy and Solar Physics]. 1983b, iss. 65, pp. 151-164. (In Russian).

Banin V.G., Borovik A.V., Yazev S.A. Complex of activity and large solar flares. Contributions of the Astronomical Observatory of Scalnation Pleso. 1986, vol. 15, pp. 289-296.

Batmunkh D., Golovko A.A., Trifonov V.D., Yazev S.A. Observations of activity complex yielded large solar flares in August 2011 in Irkutsk and Ulanbaatar. Izbrannye problemy astronomii: materialy 3 Vserossiiskoi astronomicheskoi konferentsii "Nebo i Zemlya" [Selected Problems of Astronomy: Proc. $3^{\text {rd }}$ Russian Conference “Sky and Earth"]. Irkutsk, 2011, pp. 75-81. (In Russian).

Bhatnagar A., Livingston W. Fundamentals of Solar Astronomy. World Scientific Publishing Co. Pte. Ltd., 2005. $445 \mathrm{p}$.

Borovik A.V., Myachin D.Yu. The spotless flare of March 16, 1986. Preflare activations of fine structure of chromospheres. Solar Phys. 2002, vol. 205, pp. 105-116. DOI: 10. 1023/A:1013859722017.

Borovik A.V., Myachin D.Yu. Structure and development of the spotless flare on March 16, 1981. Geomagnetism and Aeronomy. 2010, vol. 50, no. 8, pp. 937-949. DOI: 10.1134/S0016793210080037.

Borovik A.V., Myachin D.Yu., Tomozov V.M. Observations of spotless flares at the Baikal Astrophysical Observatory and their interpretation. Izvestiya Irkutskogo gosudarstvennogo universiteta. [Transactions of the Irkutsk State University]. 2014, vol. 7, pp. 23-45. (In Russian).

Evans J.W. The birefringent filter. J. Opt. Soc. Am. 1949, vol. 39, pp. 229-242.

Golovko A.A., Golubeva E.M., Grechnev V.V., Myachin D. Yu., Trifonov V.D., Khlystova A.I. Data base of full solar disk H-alpha images from the Baikal Observatory. Solar Variability: From Core to Outer Frontiers. Proc. $10^{\text {th }}$ European Solar Physics Meeting. Prague, Czech Republic, 9-14 September 2002, pp. 929-932. 
Klevtsov Yu.A. Features of working birefringent filter in telescope optical scheme Issledovaniya po geomagnetizmu, aeronomii i fizike Solntsa [Research on Geomagnetism, Aeronomy and Solar Physics]. 1984, iss. 69, pp. 183-183 (In Russian).

Klevtsov Yu.A. Novye opticheskie sistemy teleskopov i metody ikh rascheta. Diss. kand. tekh. nauk [New Optical Systems of Telescopes and Methods of Their Calculation. Cand. Tekh. Sci. Diss.]. Irkutsk, 1987, 233 p. (In Russian).

Klevtsov Yu.A., Trifonov V.D. Optical system of a new chromospheric telescope. Issledovaniya po geomagnetizmu, aeronomii i fizike Solntsa [Research on Geomagnetism, Aeronomy and Solar Physics]. 1980, iss. 52, pp. 71-75. (In Russian).

Konyaev P.A., Borovik A.V., Zhdanov A.A. Spotless flares structure and development analysis using digital images of solar chromosphere. Optika atmosfery i okeana [Atmospheric and Oceanic Optics]. 2015, vol. 28, no. 9 (320), pp. 844-849. (In Russian).

Kushtal' G.I., Skomorovsky V.I. The two-bandpass tunable birefringent filter (BF) for the HeI $10830 \AA$ and $\mathrm{H} \alpha$ lines. Opticheskiy zhurnal [Journal of Optical Technology]. 2000, vol. 67, iss. 6, pp. 99-106. (In Russian).

Kushtal G.I., Skomorovsky V.I. Advancements in the geometrical measurements of the birefringent filter's crystal plates and two-dimensional measurements of Doppler velocity in the solar atmosphere. Proc. SPIE. 2002, vol. 4900, pp. 504-512.

Öhman Y. On some new birefringent filters for solar research. Arkiv för Astronomi. 1958, vol. 2, pp. 165-169.

Proshin V.A., Aleksandrovich S.V. Sealing of dielectric filters in vacuum. Issledovaniya po geomagnetizmu, aeronomii i fizike Solntsa [Research on Geomagnetism, Aeronomy and Solar Physics]. 1982, iss.60, pp. 76-80. (In Russian).

Severnyy A.B., Gil'varg A.B. The birefringent filter for solar research and experience of its application. Izvestiya KrAO [Transactions of Crimean Astrophysical Observatory]. 1949, vol. 4, pp. 3-22. (In Russian).

Skomorovsky V.I. Examination of two birefringent filters. Rezul'taty nablyudeniy i issledovaniy v period MGSS [Results of Observations and Studies During International Year of Quiet Sun]. 1967, iss. 4, pp. 105-109. (In Russian).

Skomorovsky V.I., Ioffe S.B. Monochromatic filters for solar observations. Issledovaniya po geomagnetizmu, aeronomii i fizike Solntsa [Research on Geomagnetism, Aeronomy and Solar Physics]. 1980, iss. 52, pp.128-149. (In Russian).

Skomorovskiy V.I. Kushtal' G.I., Lopteva L.S., Proshin V.A., Tsayukova A.G. Commercial narrowband FabryPerot solar filters, methods and instruments for their examination. Solnechno-zemnaya fizika [Solar-Terrestrial Physics]. 2015, vol. 1, iss. 3, pp. 72-90. DOI: 10.12737/10537. (In Russian).

URL: ftp://ftp.iszf.irk.ru/h_alpha/ (accessed December 8, 2015).

URL: http://www.nso.edu/IAU-Com12/resources (accessed December 7, 2015).

URL: http:// archives.ru/documents/methodics/obzor_ restore-text-archival-document.shtml (accessed November 23, 2015).

URL: http://www.hida.kyoto-u.ac.jp/SMART/ (accessed November 17, 2015). 